\title{
Profesores Nóveles de Enseñanza Básica: Dilemas, Estrategias y Obstáculos para Abordar los Desafíos de una Educación Inclusiva
}

\section{Beginning Teachers of Primary Education: Dilemmas, Strategies and Obstacles to Face the Challenges of an Inclusive Education}

\author{
Tatiana Cisternas León * \\ Amparo Lobos Gormaz \\ Universidad Alberto Hurtado, Chile
}

La inserción profesional involucra múltiples desafíos para los docentes y ha sido descrita como una etapa crítica, de múltiples aprendizajes y reflexiones. Investigaciones dan cuenta de una creciente preocupación entre los docentes nóveles por cómo enfrentar la enseñanza en aulas heterogéneas y las demandas de una educación inclusiva. Esta investigación analiza las dificultades, dilemas, estrategias y obstáculos para responder a la diversidad percibidos por docentes de educación básica durante sus primeros años de iniciación. A través de un enfoque cualitativo y un diseño de estudio de casos, se recoge la experiencia de 10 docentes egresados de cuatro universidades e insertos en variados contextos escolares. Los hallazgos muestran un interés genuino por desarrollar estrategias que flexibilicen las actividades, los recursos y la evaluación para ofrecer oportunidades a todos. Sin embargo, este interés está marcado por el dilema de conjugar lo individual y lo colectivo y la necesidad de implementar una evaluación "equitativa”. Las estrategias declaradas ajustan tanto la organización y ambiente del aula como decisiones curriculares y didácticas. Los obstaculizadores que identifican están asociados a culturas escolares que estandarizan sus prácticas de enseñanza, clasifican y promueven la competencia entre estudiantes. Se concluye la relevancia de comprender la experiencia de los docentes desde las múltiples dimensiones involucradas en los procesos de cambio hacia el desarrollo de escuelas más inclusivas.

Descriptores: Profesores noveles; Enseñanza básica; Educación inclusiva; Inserción profesional; Cultura escolar.

Initiation in beginning teachers involves multiple challenges for teachers and has been described as a critical stage of multiple learning and reflections. Research reveals a growing concern among junior teachers about how to deal with heterogeneous classroom teaching and the demands of inclusive education. This research analyzes the difficulties, dilemmas, strategies and obstacles to respond to the diversity perceived by primary school teachers during their first years of initiation. Through a qualitative approach and a case study design, the experience of 10 teachers graduated from four universities working in various school contexts is collected. The findings show a genuine interest in developing strategies that make activities, resources and evaluation more flexible to offer opportunities for all. However, this interest is marked by the dilemma of combining the individual and the collective and the need to implement a fair evaluation. The declared strategies adjust both the organization and classroom environment as well as curricular and methodological decisions. The obstacles that they identify are associated with school cultures that standardize their teaching practices, classify and promote competition among students. The study concludes the relevance of understanding the experience

*Contacto: tcistern@uahurtado.cl

Recibido: 03/01/2019

$1^{\text {a }}$ Evaluación: 22/01/2019

ISSN: 0718-7378

Aceptado: 07/03/2019

www.rinace.net/rlei/ 
of teachers from the multiple dimensions involved in the processes of change towards the development of more inclusive schools.

Keywords: Beginning teachers; Primary education; Inclusive education; Professional induction; School culture.

\section{Introducción}

Los sistemas educativos han impulsado importantes transformaciones en el marco de los desafíos para avanzar hacia una educación inclusiva. Los propósitos de equidad siguen estando vigentes y podría decirse que, con mayor fuerza en el marco de movimientos migratorios, renovaciones curriculares, demandas por un mayor desarrollo de competencias ciudadanas y diversas fuerzas sociales que empujan en favor del derecho a una educación pertinente y de calidad para todas las personas, cuestionando prácticas de segregación, racismo y discriminación en la escuela.

Para impulsar y sostener estas transformaciones existe acuerdo sobre el rol protagónico que tienen los docentes. Una cultura escolar que reconozca y valore la diversidad como ventaja pedagógica requiere que docentes de enseñanza básica y media pongan en acción un conjunto de capacidades y actitudes vinculadas a la enseñanza, la evaluación, las interacciones y un ambiente de colaboración y aceptación dentro de la sala de clases.

Estas necesidades están conectadas y se sitúan en el contexto de políticas educativas que impulsan nuevas prácticas y roles de los docentes para responder a dichos desafíos. En Chile estas demandas se reflejan en nuevos marcos legales tales como la Ley de Inclusión Escolar, que busca generar condiciones para el avance hacia un sistema educacional más inclusivo a través de la eliminación de la selección en los procesos de admisión a fin de favorecer una distribución más heterogénea de la población escolar en los establecimientos educacionales. Asimismo, la ley establece la necesidad de que todos los establecimientos desarrollen planes de apoyo a la inclusión. Por otra parte, las escuelas reciben orientaciones para implementar los Programas de Integración Escolar (Decreto SN 170) en donde se ha puesto en relieve la importancia del trabajo colaborativo y la co-enseñanza (Mineduc, 2013) y más recientemente, a través del Decreto 83 los docentes están impulsados a tomar decisiones curriculares más contextualizadas y, a través de la diversificación, desarrollar variadas alternativas para abordar el currículo. Todos estos cambios tienen importantes consecuencias sobre la mirada y las capacidades docentes para el trabajo en aulas heterogéneas, los que se acentúan en un escenario con políticas educativas que envían señales contradictorias respecto de los principios de una educación inclusiva (Apablaza, 2014; Cisternas, Alegría y Alarcón, 2013; Muñoz, López y Assaél, 2015).

En efecto, en múltiples investigaciones y consultas a docentes se levanta como una de las principales preocupaciones el trabajo en aulas heterogéneas, la adaptación de la enseñanza y las formas de responder a niños, niñas y adolescentes que demandan otros apoyos o se encuentran en riesgo exclusión ¿Cómo hacer para que todos participen y aprendan? es una inquietud transversal, que se repite en docentes con distintas trayectorias y experiencias profesionales. Estudiantes de pedagogía, profesores nóveles y docentes con vasta experiencia buscan respuestas en esta dirección. 
En este contexto, la presente investigación focaliza su interés en profesoras y profesores de enseñanza básica que se inician en la profesión. Docentes nóveles o principiantes ${ }^{1}$ dan cuenta del modo en que experimentan los desafíos de avanzar hacia escuelas más inclusivas. Si bien en los últimos veinte años la investigación sobre este actor en particular ha tenido un importante desarrollo, contamos con menos evidencias que permitan comprender cómo viven estos procesos los docentes que se inician en la profesión.

Desde estos elementos se levanta la necesidad de indagar en aspectos que están a la base del escenario que se plantea hoy en relación a cómo dan respuesta a la diversidad en el aula los profesores nóveles de educación Básica. Comprender las perspectivas docentes, implica indagar en las tensiones que en un escenario como este se desprenden.

En este artículo se presenta una parte de los resultados de un estudio más amplio² cuyo propósito fue comprender los procesos de inserción a la docencia y los efectos de los contextos escolares sobre la experiencia de profesores principiantes de enseñanza básica. Los objetivos que orientaron los resultados que a continuación se exponen fueron: caracterizar dificultades que reconocen los docentes noveles para abordar la diversidad en el aula y los actuales desafíos en materia de inclusión; reconocer las estrategias que utilizan en la sala de clases para abordar dichas dificultades e identificar los obstaculizadores que experimentan en sus contextos escolares para enfrentar estos procesos.

\section{Revisión de la literatura}

La iniciación profesional de los docentes, es decir, su ingreso al mundo laboral luego de su formación inicial, se caracteriza por ser "un periodo de tensiones y aprendizajes intensivos en contextos generalmente desconocidos y durante el cual los profesores principiantes deben adquirir conocimiento profesional además de conseguir mantener un cierto equilibrio personal" (Marcelo, 2009, p. 5). Asimismo, es un período relevante en la trayectoria de los docentes por cuanto constituye un eslabón fundamental del desarrollo profesional y profundizar en éste aporta a la formación inicial y continua y a la formulación de programas y políticas de apoyo a los procesos de inserción (Ávalos, Carlson y Aylwin, 2004; Borko, 2004; Cornejo 1999; Day 2008, 2012; Feiman-Nemser 2001; Flores, 2008; Fuentealba, 2006; Ingersoll y Strong, 2011; Marcelo, 2008, 2009; Tynjälä y Heikkinen, 2011).

El conjunto de la investigación sobre los primeros años de la enseñanza se ha orientado en torno a cuatro ámbitos: i) los dilemas y conflictos que experimentan los profesores principiantes, ii) los procesos de socialización que subyacen a estos inicios; iii) la configuración de la identidad profesional; y iv) los apoyos formales e informales que recibe un docente novato (Flores, 2008). Asimismo, la investigación sobre docentes nóveles o principiantes señala que los inicios están fuertemente vinculados a las características de la formación inicial, características de los contextos escolares donde se inserta, los apoyos y

\footnotetext{
1 Si bien la literatura especializada establece algunas distinciones, en este artículo se utiliza de manera indistinta la denominación "novel" y "principiante" para referirse a los docentes que se encuentran dentro de sus tres primeros años de iniciación profesional.

${ }^{2}$ Fondecyt de Iniciación $\mathrm{N}^{\circ} 11130279$ : Diferencias en los procesos de inserción a la docencia en escuelas chilenas. Los efectos de distintos contextos escolares de iniciación sobre prácticas de enseñanza, incidentes críticos y estrategias en profesores principiantes. Se agradece el apoyo al Programa FONDECYT.
} 
las actitudes, condiciones personales y biográficas del propio docente (Cornejo, 1999; Day y Gu, 2012; Feiman-Nemser, 200 1; Flores, 2008; Fuentealba, 2006; Marcelo, 1988, 2008).

La revisión de la literatura nacional e internacional acerca de los docentes nóveles y/o profesores principiantes muestra la importancia de investigar acerca de los dilemas y preocupaciones sobre la enseñanza que experimentan los docentes los primeros años, (Ávalos, 2009; Cisternas, 2016; Ruffinelli, 2014; Ruffinelli, Cisternas y Córdova, 2017). El fenómeno denominado como choque con la realidad se expresa en dificultades, preocupaciones y dilemas de diversa naturaleza. Algunos se asocian a la relación con la organización escolar, relación con colegas, directivos, apoderados. Otro grupo de dificultades se vincula las decisiones pedagógicas, curriculares y didácticas: contenidos, materiales, organización de la clase, gestión de los alumnos, etc. (Marcelo, 1988; Vonk y Schras, 1987). Este último ámbito ha comenzado a ser estudiado más recientemente con foco en dilemas, estrategias de abordaje e instancias de apoyo vinculadas a las tareas enseñanza-aprendizaje en el aula. Más aún, pasados los primeros meses de inserción, los problemas que viven los principiantes se concentran en el campo de las prácticas de enseñanza (Agüero, 2015; Day, 2008; Flores, 2014; Lam y Yan, 2011; Long, Conwaya y Murphya, 2012; Tynjälä y Heikkinen, 2011; Rufinelli, 2014)

En Chile contamos con algunas investigaciones sobre estudiantes en práctica profesional y profesores principiantes que muestran coincidencia en las dificultades vividas en la tarea de enseñar, y las dimensiones en que perciben un menor aporte de la formación inicial. Dichos estudios entregan pistas importantes en la medida que señalan la poca contribución que tuvo la formación inicial para resolver situaciones muy típicas de la enseñanza, por ejemplo, diseñar un instrumento de evaluación que dé cuenta de la diversidad de aprendizajes en el aula (Cisternas, 2016). En suma, contamos con menos investigaciones que se refieran específicamente a las dificultades en el ámbito didáctico que experimentan cuando se enfrentar a la tarea de responder a la diversidad en el aula, es decir, aquellos dilemas que provienen específicamente del proceso de enseñanzaaprendizaje en el aula y desde el "choque" que experimentan en el plano de la enseñanza.

Respecto a las capacidades y desafíos que enfrentan los docentes para responder a la diversidad, la literatura muestra cómo se han visto modificados los roles, las funciones, el lugar y ámbito de intervención de los docentes. Cambios sistemáticos que con los años ha provocado también dilemas, dificultades y nuevas necesidades de formación que permitan dar respuesta a la nueva identidad del profesor. Desde esta perspectiva, la diversidad y las dificultades del aprendizaje son producto de las interacciones entre sujeto y la escuela, es decir, con las condiciones de escolarización. De esta manera se traslada la responsabilidad a la escuela y las prácticas de enseñanza pues desde este enfoque el foco está puesto en las barreras del sistema educativo. El cambio de mirada y el avance que han experimentado los sistemas educativos respecto de las diferencias en los aprendizajes y las distintas capacidades de los estudiantes han afectado el rol de los docentes de enseñanza básica y media (Duk, 2014; Echeita, 2006; Infante, 2010; López, Echeita y Martín, 2010; Ramos, 2013).

Investigaciones nacionales que describen las dificultades de los docentes nóveles muestran que éstas suelen estar vinculadas a la enseñanza en aulas heterogéneas y a las características de las políticas educativas y del contexto escolar (Apablaza, 2014). Un estudio reciente que compara las dificultades en docentes que se encuentran en su primer, segundo y tercer año de inserción. Destaca la semejanza entre docentes respecto a las 
dificultades que perciben ante el manejo de actividades y recursos de aprendizaje, tales como conducir actividades de aprendizaje variadas y de diferente nivel de complejidad, usar recursos didácticos variados e instrumentos de evaluación, así como manejo de contenidos; además, ellos manifiestan tener problemas para usar recursos didácticos y evaluativos diferenciados, acordes a las características de sus estudiantes. (Solís et al., 2016). Ávalos, Carlson y Aylwin (2004) detectaron que las principales dificultades en el período inicial de ejercicio docente se relacionaban con el manejo pedagógico de los contenidos de enseñanza y la atención a las diferencias individuales; aparece el cansancio físico; el manejo de las reglas y rutinas escolares; la dificultad con la disciplina y los efectos de políticas y reforma. Hallazgos que coinciden con un estudio que se realiza un seguimiento durante tres años a docentes principiantes de educación básica (Ruffinelli, Cisternas y Córdoba, 2017).

Jiménez y Montecinos (2018) examinan los enfoques con que ocho profesores novatos gestionan la diversidad escolar. Concluyen que éstos se presentan demandas directas, entre las que se cuenta el desarrollo de estrategias diversificadas de enseñanza y evaluación, donde predomina un enfoque que atribuye las dificultades de los estudiantes a déficit propios con una débil focalización en las barreras institucionales hacia el aprendizaje y la participación de sus alumnos.

El desafío que hoy enfrentan los sistemas educativos da cuenta de la necesidad de avanzar en la promoción de escuelas inclusivas que logren otorgar respuestas pertinentes a la diversidad de sus estudiantes que acceden a la educación. Ello basado en un principio de justicia e equidad en el cual se pueda garantizar una educación de calidad de la cual se beneficien todos, sin ningún tipo exclusión (Ainscow, Booth y Dyson, 2006; Echeita, 2008; Marchesi, Blanco y Hernández, 2014; OEI, 2010; UNESCO, 2005).

En los últimos años se han construido algunos consensos respecto a las capacidades que deben poner en acción los docentes desde un enfoque inclusivo: la valoración de la diversidad y comprenderlas como un recurso pedagógico, el apoyo a todos los estudiantes mediante la flexibilización del currículo, el trabajo colaborativo con distintos actores de la comunidad educativa y la capacidad para reflexionar y aprender a lo largo de la trayectoria profesional (Agencia Europea para el Desarrollo de la Educación del Alumnado con Necesidades Educativas Especiales, 2012).

En este escenario, cabe preguntarse por la visión de docentes nóveles de enseñanza básica especialmente en un escenario educativo que ha sido descrito por sus contradicciones y renovados desafíos y exigencias a la labor pedagógica.

\section{Método}

La investigación se desarrolla desde un enfoque cualitativo pues busca indagar en la naturaleza de los procesos, la subjetividad de los actores y las interrelaciones de éstas con los contextos donde se produce el fenómeno (Flick, 2004). Los propósitos se abordan mediante un diseño de estudio de casos múltiple (Stake, 1998) en combinación con algunas pautas analíticas del enfoque Teoría Fundamentada (Cisternas, 2017; Strauss y Corbin, 2002). Para la selección de los casos se utilizó el criterio de máxima variación de la muestra con el fin de asegurar heterogeneidad y profundidad en el acercamiento al fenómeno. De este modo, los participantes fueron 10 profesores nóveles seleccionados de acuerdo los siguientes criterios: a) se encuentren dentro de los dos primeros años de inserción; b) 
pertenecen a 2 regiones del país: Santiago y Valparaíso; c) realizaron estudios en instituciones de educación superior con distintos niveles de selectividad; y d) se desempeñen en diversos contextos escolares de iniciación. Esto se materializó en la selección de docentes que trabajarán en distintas dependencias: municipal, particular, particular subvencionado.

Como estrategia de recolección de información se utiliza la "entrevista episódica". Instrumento semiestructurado cuya idea de base es que las experiencias de los sujetos se almacenan y recuerdan en la forma de un conocimiento de tipo narrativo-episódico y de tipo semántico (Flick, 2000). El análisis se realiza siguiendo los principios de la teoría fundamentada. Combinando procedimientos inductivos y deductivos, por medio de la construcción y análisis de categorías y la comparación teórica constante. Para el análisis se utilizó el programa Atlas.ti. Se compararon los datos a nivel de intra-casos y entrecasos con el objetivo de descubrir patrones y relaciones en torno al fenómeno central que se está indagando (Cisternas, 2017).

\section{Resultados}

Se organizan los resultados en tres partes de acuerdo a las dimensiones estudiadas: dificultades y dilemas para abordar la diversidad en el aula percibidas por los docentes noveles de enseñanza básica, estrategias que señalan utilizar para responder a los desafíos de aulas heterogéneas y obstaculizadores o barreras para hacer frente a éstos.

\subsection{Dificultades y dilemas para abordar la diversidad en el aula}

¿Cuáles son las preocupaciones y dilemas de los docentes nóveles respecto al abordaje de la en sus prácticas cotidianas de enseñanza? Comprender esta dimensión da luces sobre sus enfoques acerca de la diversidad y las diferencias, las capacidades y saberes que poseen y las que requieren mayor desarrollo para abordar estos desafíos desde la perspectiva de la educación inclusiva (figura 1).

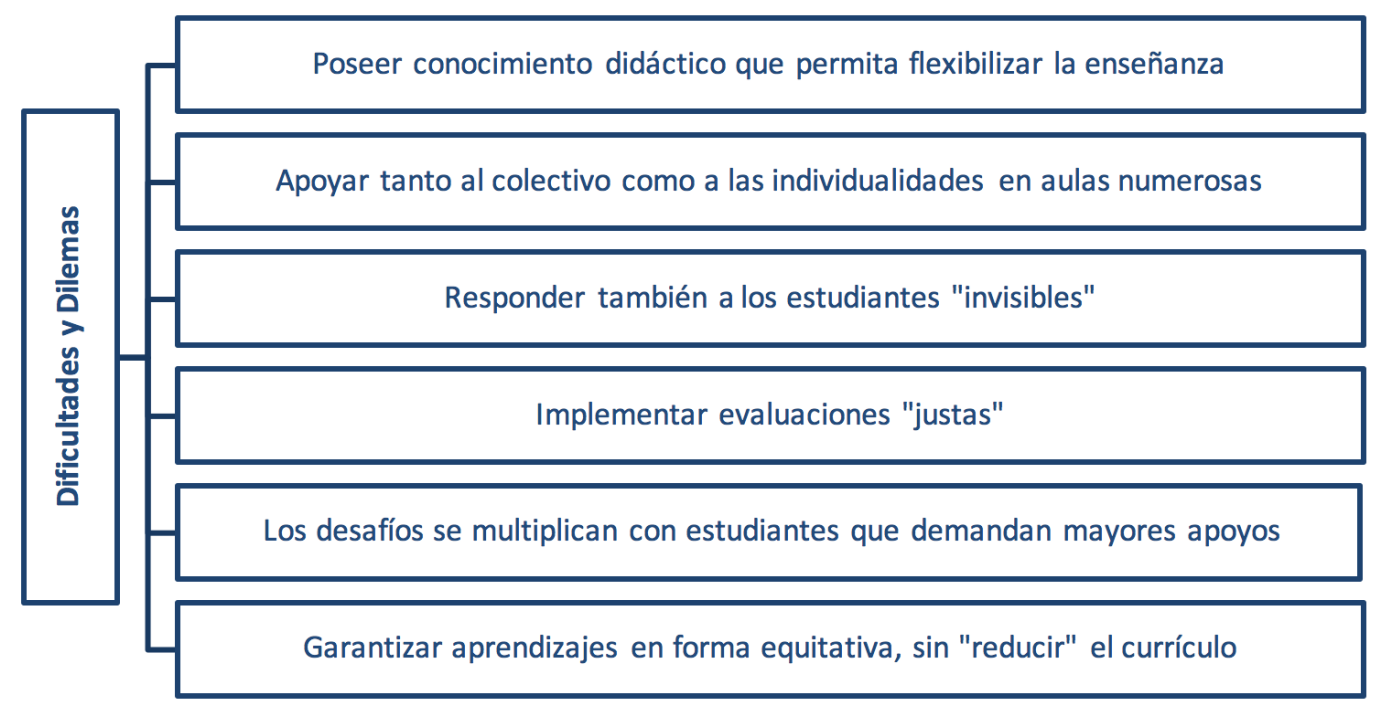

Figura 1. Dificultades y dilemas para enseñar en aulas diversas Fuente: Elaboración propia. 
Un primer aspecto que preocupa a los docentes es la dificultad para poner en acción un repertorio variado de estrategias de enseñanza que asegure oportunidades de aprendizaje a todos sus estudiantes incluyendo aquellos que demandan mayores apoyos. Esto, señalan, implica poseer un mayor conocimiento didáctico de las áreas específicas de enseñanza. Por ello, les resulta difícil el diseño e implementación de prácticas donde puedan ir más allá de una única forma de abordar un objetivo de aprendizaje. Un manejo más profundo de las facetas de los contenidos curriculares y las distintas maneras de presentarlo contribuiría a una práctica pedagógica más flexible y variada.

Una segunda preocupación transversalmente sentida por las docentes es la búsqueda de un equilibrio entre las demandas colectivas de sus estudiantes y los apoyos individualizados a quienes lo requieran. ¿Cómo puedo focalizar mis apoyos en un aula numerosa?, ¿cómo ofrecer ayuda a un grupo pequeño sin desatender a los demás? Esto provoca una tensión especialmente en situaciones de monitoreo y retroalimentación durante la clase. Los docentes perciben que en la mayoría de las ocasiones no pueden "tomar en cuenta" a aquellos estudiantes que experimentan más dificultades. Sostienen que esto resulta un problema pues las demandas de un aula numerosa superan sus posibilidades para entregar apoyos focalizados. Además, experimentan un conflicto adicional: el prestar este tipo de ayudas individualizadas puede generar diferencias no deseadas entre sus estudiantes.

\section{(...) Entonces ¿̨cómo lo hago? lo único que yo puedo hacer con ellos es estar pendiente de ellos; o sea, pasar por ellos y preguntarles cómo van, cómo estamos. Esto se hace así, esto se hace acá. Pero si tú me dices que les dedique el tiempo a ellos no puedo. El tiempo que les tengo que dedicar a ellos no se los puedo dar, y tiene que ver netamente con el número de alumnos. (Profesora Blanca)}

Similar al punto anterior, otra dificultad asociada a la diversidad en el aula es la deuda que tienen con los estudiantes más "silenciosos". La gestión de la clase suele estar mucho más demandada por niños y niñas que por distintas razones atraen la atención y los esfuerzos del profesor (dentro de este grupo describen a los estudiantes más activos y desatentos y aquellos con diagnósticos de autismo o asperger). En ese contexto, dicen no saber cómo responder oportunamente a esos estudiantes “invisibles". Niños y niñas que no se ven, ya sea en sus avances o barreras para lograr los aprendizajes, y que, a juicio de ellos, muchas veces pasan a un segundo plano dentro del sinnúmero de tareas que deben cumplir dentro del aula.

Me preocupa que los silenciosos tienden a pasar muy desapercibidos para mí y los bulliciosos, escandalosos se me va la vida entera... Me preocupa como poder abordarlos (...) porque no sé cómo, solamente tengo la prueba para medir sus aprendizajes, no sé si me entendieron porque no me preguntan. No sé tanto de sus vidas, al final es un grupo de niños que uno proyecta que los va a ver más o menos seguidos (...) Me pregunto si estarán aprendiendo. (Profesora Paloma)

Una cuarta dificultad en sus prácticas de enseñanza es la de diseñar e implementar evaluaciones que sean "justas", es decir, que entreguen información relevante acerca del progreso en el aprendizaje de cada uno de sus estudiantes y también le sean útiles para la toma de decisiones. La preocupación se sitúa especialmente en estudiantes cuyas capacidades tienen un mayor desfase en relación al nivel de escolaridad y donde "la prueba les quedará grande.” Los docentes entrevistados se preguntan cómo diferenciar sus instrumentos de evaluación, pero evitando la reducción y simplificación de los ítems. Algunos aluden a la falta de conocimientos sobre otras estrategias de evaluación que, por 
ejemplo, vayan más allá de la modificación a la escala de evaluación, práctica que sabemos resulta muy habitual ante escenarios como éste:

\begin{abstract}
"Me complica hacer actividades distintas, por un dar un ejemplo: la misma actividad de escribir una narración, lo mismo solo que quizás sea más breve, quizás se demore más, porque también los niños se ven mucho en sus pares, entonces distanciarlo tanto y decirle 'para usted es esta guía distinta' no me gusta. $\Upsilon$ ya es incómodo porque me quedo sin respuesta si me dicen '¿̈por qué a él le está preguntando otra cosa?' o 'tía, usted dijo que no le iba a ayudar a nadie' y les contesto es que a la Sofía tengo que ayudarla... (Profesora Sara)
\end{abstract}

En esta línea, entre los docentes se observaron dificultades de distinta naturaleza para asegurar que sus evaluaciones sean útiles y a la vez justas. Para algunos el desafío está en implementar evaluaciones que consideran a sus estudiantes con mayores dificultades sin hacer diferencias en los instrumentos y actividades de evaluación y evitando reducir la complejidad de la tarea y su exigencia. Para ellos resulta importante no distanciar el nivel de exigencia de los estudiantes respecto del resto de sus compañeros y sin embargo les resulta difícil no hacerlo. Para otros, la mayor dificultad tras la flexibilización de la evaluación se asocia a los efectos sobre el resto de los compañeros de clase y las reacciones que surgen cuando el ajuste en la evaluación para algunos aparece como un "privilegio" que rechazan. Se trata de docentes a quienes les parece importante evitar posibles conflictos o situaciones de discriminación por los compañeros que no son beneficiados por este tipo de evaluación.

(...) lo único institucional que había en el colegio era la evaluación diferenciada, te
decían a ti que adecuaras la evaluación y tú te preguntas ¿̇cómo lo hago? Primero hice
reducción de items, cambié la letra, eran cosas más de acceso a la evaluación del
contenido: a lo mejor a este niño que dibuja mejor, hacerlo que me dibuje o que te lo
explique con palabras o diagramas, cosas así (...) a cada profesor se le pedía que
adecuara su prueba. To hacía la fila A, la fila B y esa era la “diferencial”. De hecho,
los niños se sentían muy identificados porque todos sabían y los otros decían: "profesor
qué injusto, para ellos es más fácil... (Profesor José)

Una quinta preocupación de los docentes nóveles se produce con la presencia en sus aulas de estudiantes que demandan mayores apoyos ya sea por una condición de discapacidad, un trastorno del comportamiento u otra situación que los ubica en situación de mayor desventaja respecto al resto. En efecto, perciben que el desafío se multiplica: ajustar el currículo para un estudiante con diagnóstico de Asperger que impacta especialmente el comportamiento y la interacción social implica decisiones diferentes ante otro estudiante cuyo diagnóstico sea de discapacidad intelectual. Un hallazgo significativo es que para los docentes nóveles la presencia de estudiantes con estas características (por ejemplo, con diagnóstico de Asperger) genera mayores conflictos. Se ponen en juego aspectos propios de la identidad profesional y la vocación o bien, emerge el cuestionamiento a la presencia en la escuela regular de niños y niñas con estas características.

Finalmente, observamos una preocupación cuyo carácter dilemático resulta evidente. Los docentes dicen no tener respuestas que les permitan garantizar aprendizajes en forma equitativa y sin "reducir" el currículo. Un aspecto que marca fuertemente la identidad de las y los docentes de educación básica es la de lograr que todos sus estudiantes accedan en forma igualitaria a aprendizajes relevantes. Es decir, cómo hacer que todos sus estudiantes aprendan lo mismo, reconociendo la diversidad de niveles y conocimientos previos. El dilema es visto de maneras distintas entre los docentes. 
(...), sino que ver que todos son distintos y al final, aunque todos sean distintos igual tienes que llegar a un aprendizaje y llegar a un concepto estándar, pero desde la forma en que cada uno de ellos trabaja. (Profesora Paulina)

Algunos plantean que esto implica aceptar que se desarrollarán aprendizajes distintos (desiguales) entre sus estudiantes y necesariamente hay que seleccionar y descartar objetivos de aprendizaje cuando sea necesario: se aprenden cosas distintas y la reducción del currículo que se aplica, en ocasiones, trae consigo una disminución en las expectativas sobre las capacidades de los estudiantes. Otros docentes hacen una lectura diferente a este dilema: aspiran a que todos los estudiantes -sin distinción por nivel de logro o habilidadesdesarrollen los mismos aprendizajes por medio de la diversificación de sus estrategias y el despliegue de una variada batería de recursos y formas de presentar un contenido en particular: se aprende lo mismo, pero de distintas maneras. El desafío está en tener la capacidad para hacerlo atendiendo no sólo a las propias competencias sino también a los obstáculos que impone la cultura escolar.

\subsection{Estrategias utilizadas por las docentes nóveles para responder a la diversidad}

Las docentes describen variadas estrategias que se distinguen por el ámbito que abarcan y que clasificamos en dos grupos. Un primer conjunto se relaciona con decisiones que afectan la organización, el ambiente de la sala de clases y las relaciones sociales y la convivencia entre estudiantes. Un segundo grupo de estrategias -y que tiene mayor presencia, relevancia y variedad-involucra decisiones sobre los procesos referidos a qué, cómo y cuándo enseñar. Es decir, de qué manera se favorecen mejores aprendizajes para todos los estudiantes, en qué tiempos y cómo éstos son evaluados (figura 2).

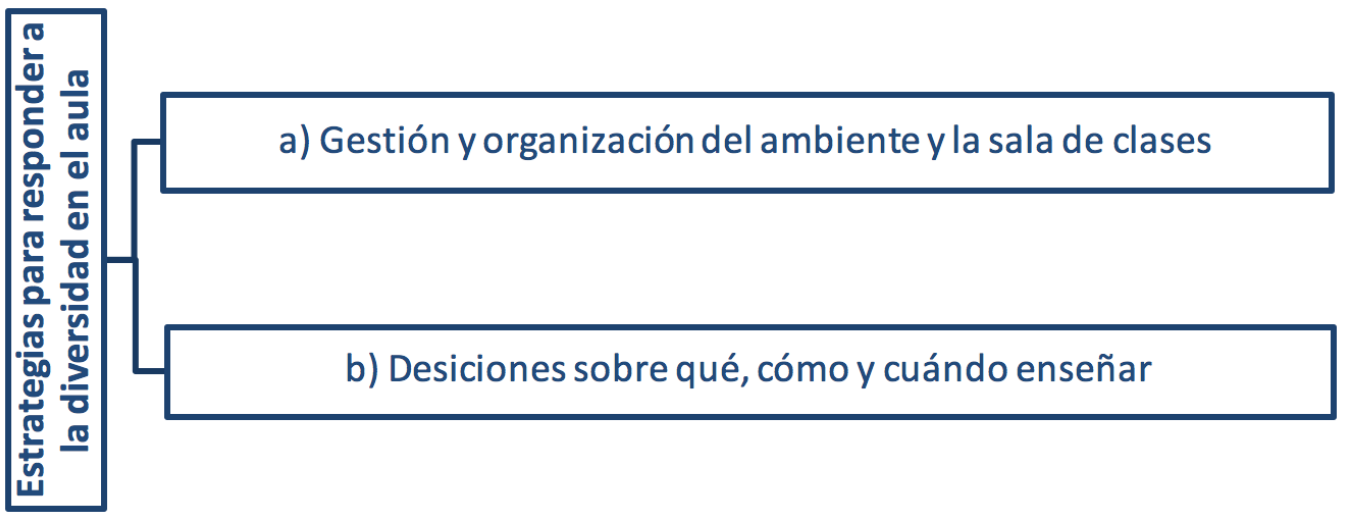

Figura 2. Estrategias para responder a la diversidad en el aula Fuente: Elaboración propia.

Respecto al primer grupo de estrategias, los docentes nóveles buscan distintas maneras para lograr un espacio donde existan condiciones que aseguren la convivencia y participación de todos sus estudiantes: a) definir la ubicación de los estudiantes en la sala, cambiarla para hacer funcionar tutorías entre pares o para ayudar a la autorregulación del comportamiento; y b) promover mayor participación y trabajo colectivo mediante juegos o competencias entre grupos de estudiantes (por filas o equipos).

Tengo estudiantes que son diagnosticados limítrofes, y el otro con discapacidad intelectual. Entonces qué hago yo, el B se sienta atrás, de los últimos, pero los más inquietos están al lado mío... entonces a los que tienen dificultades para aprender igual van juntos, o sea como la E con la F, que ambas tienen el mismo diagnóstico 
entonces están las dos sentadas juntas porque si la siento con otros son molestosos. (Profesora Javiera)

“(...) los niños que tienen problemas o menor rendimiento, los siento como los mejores y que los mejores también se hacen cargo de ayudar a sus compañeros, yo les digo: no le den la respuesta, pero ayúdalo a pensar, porque entre pares se entienden mejor a veces. (Profesora Marlene)

Un segundo grupo de estrategias se asocia a decisiones sobre qué y cómo promover aprendizajes en todos los estudiantes. Cabe señalar que ante esta dimensión los docentes describen estrategias dirigidas principalmente a estudiantes que demandan mayores apoyos y/o son parte del Programa de Integración Escolar. Identificamos al menos tres tipos de acciones: a) considerar los conocimientos previos, estilos de aprendizaje e intereses especialmente para diseñar y ajustar sus actividades y generar situaciones contextualizadas a la vida cotidiana de sus estudiantes; b) incorporar otros recursos para la enseñanza, principalmente material concreto; y c) evaluar de manera diferenciada, siendo esta última la estrategia con mayor prevalencia entre los docentes.

(...) considerar qué es lo que los niños saben, creo que hay que partir por ahí, qué es lo que ellos manejan de los contenidos, de qué forma ellos los abordan, cómo plantearles una situación y de qué forma ellos van a lograr desarrollar esa situación, preguntarme qué es lo que pretendo llegar con eso. (Profesora Paulina)

(...) En Matemáticas hay niños que ya están en un nivel más abstracto y a los niños que les cuesta más, les ponemos el material concreto. Con esos niños trabajamos en grupo y los que ya pueden resolver la guía trabajan solos, entonces con los niños que les cuesta más tomamos el material concreto y empezamos a repartir y vamos comparando. (Profesora Marlene)

La evaluación es un tema donde también surgen matices. Para unos docentes resulta importante hacer diferencias en los procesos y procedimientos para evaluar al curso y a los estudiantes con dificultades. Hay otros docentes en cambio, que plantean una evaluación común en la que se consideran los distintos niveles de ejecución de los niños y sus estilos de aprendizaje.

Cuando se refieren a la manera de evaluar a aquellos estudiantes que experimentan mayores dificultades, vemos que utilizan la "evaluación diferenciada", entendida esta como la modificación de estructura y contenido de las evaluaciones que se aplican a todos los estudiantes. Así, describieron una variedad de ajustes: cambiar y ampliar la letra, flexibilizar la respuesta de acuerdo a los intereses, reducir o modificar los objetivos de aprendizaje y reducir o eliminar ítems.

\subsection{Obstaculizadores para responder a la diversidad en el aula}

Esta investigación analizó la mirada de los docentes noveles respecto a las barreras que identifican para responder a las dificultades y estrategias que ponen en acción en el contexto de su trabajo en aulas diversas. Identificamos dos tipos de obstaculizadores (figura 3).

Todos los docentes nóveles sostienen que se insertaron en espacios escolares donde las culturas, normativas y definiciones institucionales les imponen barreras para las prácticas que quisieran desarrollar. Destacan tres obstáculos: a) se promueve la competencia entre estudiantes y su clasificación y ordenamiento según rendimiento, b) existe presión por lograr cobertura curricular, aunque el costo sea la falta de profundización y c) la tendencia a estandarizar las estrategias de enseñanza y/o de evaluación. 


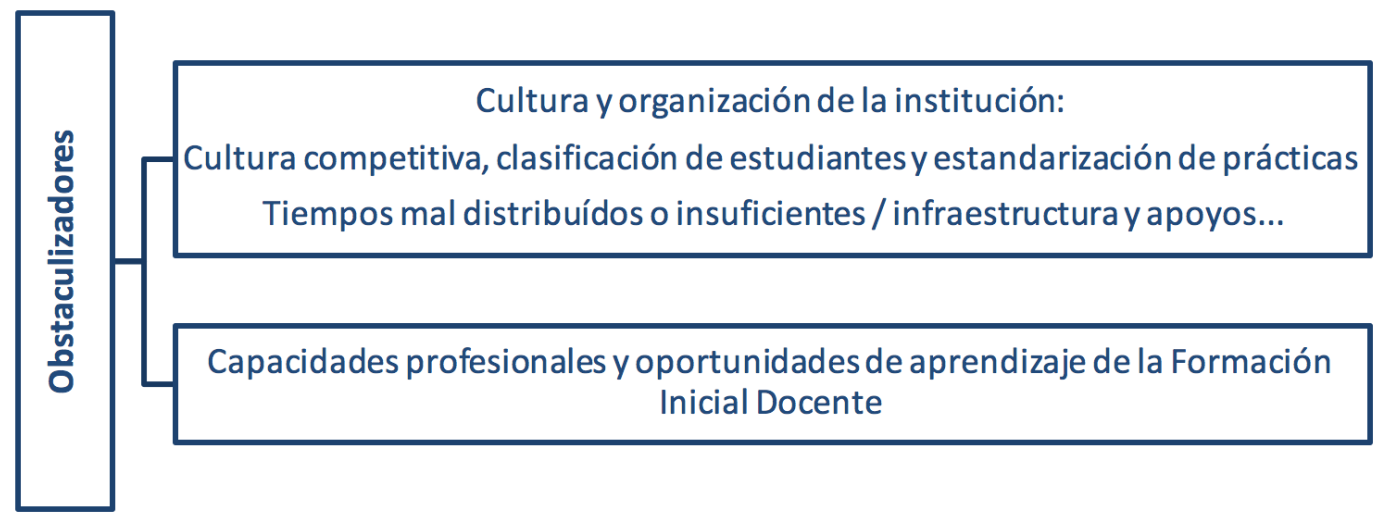

Figura 3. Obstaculizadores para responder a la diversidad en el aula Fuente: Elaboración propia.

La organización escolar privilegia modalidades que refuerzan las diferencias y la segregación entre los niños y les pide distinguir (evitando "mezclar") a quienes experimentan dificultades de los que no con el fin de focalizar mejor los apoyos. Distinto a la idea de algunos docentes que consideran que el trabajo en grupos heterogéneos beneficia los procesos de aprendizaje de los estudiantes en su conjunto.

\begin{abstract}
Tengo un conflicto porque a veces dicen que hay que tener identificados los niños que son 'insuficientes', adecuando, elementales y en el colegio nos dicen que tenemos que separarlos en filas en la sala, pero considero que entre todos mezclados se pueden apoyar mejor que tenerlos segmentados, estar preocupándonos de una sola fila y despreocuparme de los que ya están bien. (Profesora Marlene)
\end{abstract}

Otro aspecto significativo y donde se refleja una tensión con la organización escolar refiere a la evaluación de los aprendizajes. Los centros suelen definir una única modalidad de "evaluación diferenciada" que implica la mantención de instrumentos de respuesta cerrada y la reducción o simplificación de ítems. Los docentes nóveles indican que la implementación de estas formas evaluativas, sin permitir otras estrategias, no informa sobre los avances de los estudiantes, sus aprendizajes y las habilidades que han desarrollado en el proceso.

\title{
Lo que me ha pasado en todos los colegios es que al final yo siento solo quitan preguntas y no hacen nada más, (...) le quitaron tales preguntas, pusieron una de alternativas, y yo no sé qué pasa detrás de ese niño, cuáles son sus avances netamente de habilidades. (Profesora Javiera)
}

Otro conjunto de obstáculos identificados refiere a las condiciones organizacionales y laborales. Estas son: a) la escasa disponibilidad de tiempo para diseñar y evaluar la enseñanza, ellos saben que se requiere pensar mejor la enseñanza en aulas heterogéneas y eso demanda más tiempo b) una infraestructura y espacios disponibles que dificultan la implementación de otros modos de organización y agrupamiento entre estudiantes; c) la relación entre cantidad de alumnos por curso y profesionales de apoyo y d) la ausencia de los apoyos profesionales (principalmente docentes de educación diferencial/especial).

En efecto, y tal como ha sido planteado por innumerables investigaciones, la falta de tiempo para destinar a la planificación colaborativa, el conocimiento más profundo de sus estudiantes, la evaluación formativa y un seguimiento cualitativo a los aprendizajes, la preparación de recursos de enseñanza, entre otras prácticas: 
Yo creo que sí porque podría aplicar otras cosas o ver otras formas para poder hacerlo. Darme el tiempo de conocer un poco más a los niños, pero me pasa que el tema del tiempo también me juega en contra. (Profesora Paulina)

Por otra parte, algunos docentes dan importancia a la infraestructura y los espacios disponibles como una barrera o un facilitador. Organizar el aula para agrupar de maneras distintas a los estudiantes u organizar rincones de trabajo se ve imposibilitado cuando los espacios son muy reducidos salas, hay hacinamiento o un mobiliario adecuado. Además, cuando el número de estudiante es mayor este aspecto resulta aún más significativo.

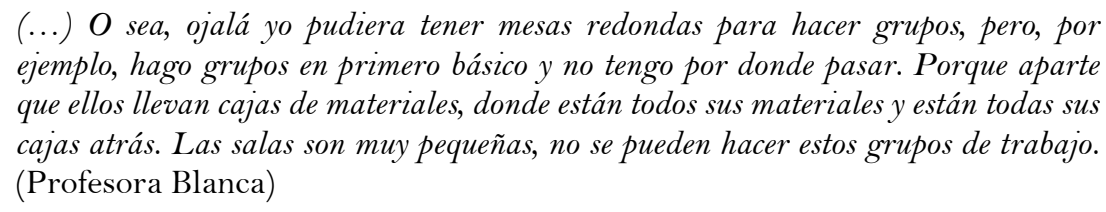

Finalmente, todos los docentes señalan la importancia de contar con la colaboración de otros profesionales y reconocen que a pesar de existir programas (como los PIE), su trabajo es más bien solitario. Cabe señalar que estos apoyos son concebidos en forma diferente por los docentes según el lugar y las funciones que esperan desarrollen los docentes de educación diferencial: Sólo para diagnosticar y trabajar ocasiones se observa fuera del aula y en otros, dentro de ella. Para otros en cambio, sería relevante contar con profesionales en procesos que van más allá del diagnóstico inicial, por ejemplo, aquellos que observan el trabajo conjunto en espacios de colaboración.

Por último, si bien el análisis da cuenta de una distancia entre lo que ocurre en las escuelas donde se insertan y lo que a juicio de ellos resultaría más provechoso y pertinente para sus estudiantes, en su mayoría adoptan una actitud de sumisión estratégica que justifican en la posición de "recién llegados".

\section{Conclusiones}

La inducción profesional como espacio protegido de apoyo y desarrollo para los profesores que se inician en la docencia es un derecho y una necesidad que con los años ha cobrado mayor relevancia a nivel nacional e internacional. Es un período crítico para la configuración de la identidad y consolidación profesional. Por ello, comprender la experiencia y visión de los docentes nóveles respecto a los desafíos actuales en materia de inclusión educativa resulta un insumo significativo para la elaboración de programas de mentoría, para la formación continua y para analizar fortalezas y debilidades de la formación inicial docente.

Los resultados de esta investigación dan cuenta de un interés genuino de los docentes nóveles por responder a los desafíos del trabajo en aulas heterogéneas, contrario a la idea de una actitud indiferente o des-responsabilizada. Este hallazgo sintoniza con otras investigaciones que refuerzan la idea de que quienes presentan menor experiencia docente pueden tener mayor sensibilidad y convicción respecto a desarrollar una enseñanza para todos los estudiantes (Granada, Pomés y Sanhueza, 2013; Izuzquiza, Echeita y Simón, 2015; Ruffinelli, Cisternas y Córdoba, 2017). Los docentes que se inician tienen un alto sentido de responsabilidad con el aprendizaje de todos sus estudiantes que en parte se explica porque el ser "buen" profesor se juega en la capacidad de movilizar a todos más allá de sus diferencias. Podría interpretarse como un claro sentido de equidad que sin embargo se da junto al reconocimiento de que esta intención les resulta difícil de concretar. 
En suma, responder a la diversidad para los docentes implica un compromiso y un desafío real.

Respecto a las dificultades percibidas por los docentes nóveles se concluye que en su mayoría aluden a desafíos propios de los procesos de cambio que provoca un enfoque inclusivo. Cuando refieren a la paradoja de responder al colectivo y también a las necesidades individuales de sus estudiantes, es posible identificar un dilema que ha sido ampliamente descrito por la literatura: "los sistemas educativos están sujetos a una doble y contradictoria exigencia. Por un lado, deben ofrecer una educación común para todos los estudiantes, pero, al mismo tiempo, deben reconocer y ajustarse a las necesidades de aprendizaje individuales de los estudiantes" (López, Echeita y Martín, 2010, p. 156). En este sentido, implementar evaluaciones "justas", ofrecer respuestas pertinentes a estudiantes que demandan mayores apoyos, o asegurar que todos participen del currículo (con el conflicto de seleccionar o descartar aprendizajes) son expresadas como dificultades que claramente se enmarcan en la naturaleza dilemática de estos procesos en tanto sus decisiones suponen consecuencias que pueden favorecer o no a todos los estudiantes. Finalmente, cabe destacar que estos hallazgos coinciden con investigaciones recientes donde se reafirma que la principal demanda en torno a la diversidad se concentra en la dimensión didáctica de la labor educativa y el peso que tiene la presión por cumplir estándares y lógicas escolares burocratizadas que agregan complejidad al trabajo docente y en la necesidad de dar un trato igualitario, evitando visibilizar en exceso las diferencias para no generar efectos negativos (Cisternas, 2016; Jiménez y Montecinos, 2018; Ruffinelli, Cisternas y Córdoba, 2017).

En cuanto a las estrategias para responder a la diversidad se concluye que los docentes noveles fluctúan entre lo necesario y lo posible. Todos los docentes declaran haber ensayado diversas estrategias para que sus estudiantes aprendan y participen de la clase, algunas ligadas al ambiente y la organización del aula y otras relacionadas a decisiones sobre el qué, cómo y cuándo enseñar. Sin embargo, similar a otras investigaciones, también reconocen una brecha entre las estrategias que quisieran implementar y las condiciones personales y de la institución escolar para avanzar en esa dirección (Lerner, 2007; Sánchez-Tarazaga, 2014). En esta línea, las barreras u obstáculos que deben enfrentar cotidianamente los docentes para avanzar hacia prácticas coherentes con un enfoque inclusivo son muchas y de diverso tipo. Culturas escolares competitivas con tendencia a la estandarización de las prácticas y pocos espacios para la reflexión al interior de la comunidad escolar, se mezclan con la autopercepción de insuficientes capacidades profesionales y una formación inicial docente que ofreció pocas o nulas oportunidades para desarrollarlas. Estos hallazgos refuerzan la necesidad de acento en las condiciones y organización del dispositivo escolar. Lo anterior se relaciona con lo que algunos autores han denominado una gramática escolar (Baquero, 2000; Terigi, 2009) o barreras para la inclusión asociadas a políticas culturas y prácticas de la escuela (Booth y Ainscow, 2002)

Finalmente, los resultados de esta investigación son relevantes para situar todos estos desafíos en el contex to de culturas escolares rígidas y burocratizadas y bajo marcos legales que suelen presentar contradicciones o incentivos distantes de los valores y propósitos de una educación inclusiva. Comprender la perspectiva de los docentes nóveles contribuye a re-situar una tendencia a individualizar -y muchas veces responsabilizar- sólo en los profesores el desafío de construir escuelas donde todos los estudiantes participen, que disfruten y progresen en sus trayectorias educativas. 


\section{Referencias}

Agencia Europea para el Desarrollo de la Educación del Alumnado con Necesidades Educativas Especiales. (2012). Formación del profesorado para la educación inclusiva. Perfil profesional del docente en la educación inclusiva. Bruselas: Agencia Europea para el Desarrollo de la Educación del Alumnado con Necesidades Educativas Especiales.

Agüero, C. (2015). Maestras principiantes y la construcción de propuestas didácticas. En R. Menghini y M. Negrin (Comps.), Docentes principiantes. Aventuras y desventuras de los inicios en la enseñanza (pp. 159-178). Buenos Aires: Noveduc.

Ainscow, M., Booth, T. y Dyson, A. (2006). Mejorando escuelas, desarrollando inclusión. Nueva York, NY: Routledge.

Apablaza, M. (2014). Representaciones sociales de profesores respecto de la diversidad escolar en relación a los contextos de desempeño profesional, prácticas y formación inicial. Estudios Pedagógicos, 4O(1), 7-24. https://doi.org/10.4067/S0718-07052014000100001

Ávalos, B. (2009). La inserción profesional de los docentes. Profesorado. Revista de Currículum y Formación de Profesorado, 13(1), 43-59.

Avalos, B., Carlson, B. y Aylwin, P. (2004). La inserción de profesores neófitos en el sistema educativo: ¿Cuánto sienten que saben y cómo perciben su capacidad docente en relación con las tareas de enseñanza asignadas? Santiago de Chile: Fondecyt.

Baquero, R. (2000). Lo habitual del fracaso y el fracaso de lo habitual. En M. Avendaño y C. Boggino (Comps.), La escuela por dentro y aprendizaje escolar (pp. 11-24). Rosario: Homo Sapiens.

Booth, T. y Ainscow, M. (2002). Guía para la evaluación y mejora de la educación inclusiva. Madrid: INTEF

Borko, H. (2004). Professional development and teacher learning: Mapping the terrain. American Educational Researcher Journal, 33(8), 3-15.

Cornejo, J. (1999). Profesores que se inician en la docencia: algunas reflexiones al respecto desde América Latina. Revista Iberoamericana de Educación, 19, 51-100.

Cisternas, T. (2016). Profesores principiantes de educación básica: Dificultades de la enseñanza en contextos escolares diversos. Estudios Pedagógicos, 42(4), 31-48. https://doi.org/10.4067/So718-07052016000500003

Cisternas, T. (2017). Desafíos y principios para investigar con teoría fundamentada. En S. Pantoja y J. Angulo (Coords.), Investigación cualitativa en educación (pp. 267-278). Buenos Aires: Miño \& Dávila.

Cisternas, T., Alegría, M. y Alarcón, P. (2013). Educación diferencial y aprendizaje escolar al debate: Repensando las dificultades. Cuaderno de Educación, 56, 13-41.

Day, C. (2008). Committed for life? Variations in teachers' work, lives and effectiveness. Journal of Educational Change, 9, 243-260. https://doi.org/10.1007/s10833-007-9054-6

Day, C. y Gu, Q. (2012). Profesores: Vidas nuevas, verdades antiguas. Una influencia decisiva en la vida de los alumnos. Madrid: Narcea.

Duk, C. (2014). La formación y el desarrollo profesional de los docentes para una educación inclusiva. En A. Marchesi, R. Blanco y L. Hernández (Coords.), Avances y desafíos de la educación inclusiva en Iberoamérica (pp. 61-70). Madrid: OEI.

Echeita, G. (2006). Educación para la inclusión o educación sin exclusiones. Madrid: Narcea. 
Echeita, G. (2008). Inclusión y exclusión educativa. Voz y quebranto. REICE. Revista Iberoamericana sobre Calidad, Eficacia y Cambio en Educación, 6(2), 3-13.

Feiman-Nemser, J. (2001). Helping novices learn to teach lessons from an exemplary support teacher. Journal of Teacher Education, 52(1), 17-30. https://doi.org/10.1177/0022487101052001003

Flick, U. (2000). Episodic interviewing. En A. M. Bauer y G. Gaskell (Eds.), Qualitative researching with text, image and sound. A handbook (pp. 75-92). Londres: Sage.

Flick, U. (2004). Introducción a la investigación cualitativa. Madrid: Morata.

Flores, A. (2008). La investigación sobre los primeros años de enseñanza: Lecturas e implicaciones. En C. Marcelo (Ed.), El profesorado principiante inserción a la docencia (pp. 59-98). Barcelona: Octaedro.

Flores, C. (2014). Inducción de profesores novatos en Chile: Un estudio de caso. Pensamiento Educativo. Revista de Investigación Educacional Latinoamericana, 51(2), 41-55 https://doi.org/10.7764/PEL.51.2.2014.4

Fuentealba, R. (2006). Desarrollo profesional docente: Un marco comprensivo para la iniciación pedagógica de los profesores principiantes. Foro Educacional, 10, 65-106.

Granada, M., Pomés, M. P. y Sanhueza, S. (2013). Actitud de los profesores hacia la inclusión educativa. Papeles de Trabajo, 25, 35-47.

Infante, M. (2010). Desafíos a la formación docente: Inclusión educativa. Estudios Pedagógicos, 36(1), 287-297. https://doi.org/10.4067/S0718-07052010000100016

Ingersoll, R. M. y Strong, M. (2011). The impact of induction and mentoring programs for beginning teachers: A critical review of the research. Review of Educational Research, 81(2), 201-233. https://doi.org/10.3102/0034654311403323

Izuzquiza, D., Echeita, G. y Simón, C. (2015). La percepción de estudiantes egresados de magisterio en la Universidad Autónoma de Madrid sobre su competencia profesional para ser profesorado inclusivo: Un estudio preliminar. Tendencias Pedagógicas, 26, 197-216.

Jiménez, F. y Montecinos, C. (2018). Diversidad, modelos de gestión y formación inicial docente: Desafíos formativos desde una perspectiva de justicia social. Revista Brasileira de Educação, 23, 1-21.

Lam, B. y Yan, H. (2011). Beginning teachers' job satisfaction: The impact of school-based factors. Teacher Development. An International Journal of Teachers' Professional Development, 15(3), 333-348.

Lerner, D. (2007, diciembre). Enseñar en la diversidad. Conferencia presentada en las Primeras jornadas de educación intercultural de la provincia de Buenos Aires. Universidad Nacional de La Plata.

Long, F., Halla, K., Conwaya, P. y Murphya, R. (2012). Novice teachers as 'invisible' learners. Teachers and Teaching: Theory and Practice, 18(6), 619-636. https://doi.org/10.1080/13540602.2012.746498

López, M., Echeita, G. y Martín, E. (2010). Dilemas en los procesos de inclusión: Explorando instrumentos para una comprensión de las concepciones educativas del profesorado. Revista Latinoamericana de Educación Inclusiva, 4(2), 155-176.

Marcelo, C. (1988). Profesores principiantes y programas de inducción a la práctica docente. Enseñanza E Teaching, 19(6), 39-54. 
Marcelo, C. (2008). Políticas de inserción a la docencia: De eslabón perdido a puente para el desarrollo profesional docente. En C. Marcelo (Ed.), El profesorado principiante inserción a la docencia (pp. 7-58). Barcelona: Octaedro.

Marcelo, C. (2009). Los comienzos en la docencia: Un profesorado con buenos principios. Profesorado. Revista de Currículum y Formación de Profesorado, 13(1), 1-25.

Marchesi, A., Blanco, R. y Hernández, L. (2014). Avances y desafíos de la educación inclusiva en Iberoamérica. Madrid: OEI.

Muñoz, M. L., López, M. y Assaél, J. (2015). Concepciones docentes para responder a la diversidad: ¿Barreras o recursos para la inclusión educativa? Psicoperspectivas, 14(3), 68-79.

OEI. (2010). Metas 2021. La educación que queremos para la generación de los bicentenarios. Madrid: OEI.

Ramos, L. (2013). Educación especial y educación inclusiva en Chile: ¿En punto de estancamiento? Revista Latinoamericana de Educación Inclusiva, 7(2), 37-46.

Ruffinelli, A. (2014). ¿Qué aprenden los docentes en su primer año de ejercicio profesional? Representaciones de los propios docentes principiantes. Pensamiento Educativo. Revista de Investigación Educacional Latinoamericana, 51(2), 56-74.

Ruffinelli, A., Cisternas, T. y Córdoba (2017). Iniciarse en la docencia. Relatos de once experiencias. Santiago de Chile: Ediciones Universidad Alberto Hurtado.

Sánchez-Tarazaga, L. (2014). Competencias docentes, atención a la diversidad e implicaciones en la formación. La voz del profesorado de Castellón. Recuperado de http://www.quadernsdigitals.net/datos_web/hemeroteca/r_77/nr_839/a_11371/11371. pdf

Solís, M., Núñez, C., Contreras, I. y Ritterhaussen, S. (2016). Inserción profesional docente: Problemas y éxitos de los profesores principiantes. Estudios Pedagógicos, 42(2), 331-342. https://doi.org/10.4067/s07 18-07052016000200019

Stake, R. (1998). Investigación con estudio de casos. Madrid: Morata.

Strauss, A. L. y Corbin, J. (2002). Bases de la investigación cualitativa: Técnicas y procedimientos para desarrollar la teoría fundamentada. Medellín: Universidad de Antioquia.

Terigi, F. (2009). Segmentación urbana y educación en América Latina. El reto de la inclusión escolar. Madrid: Fundación Iberoamericana para la Educación, la Ciencia y la Cultura.

Tynjälä, P. y Heikkinen, H. L. (2011). Beginning teachers' transition from pre-service education to working life. Zeitschrift für Erziehungswissenschaft, 14(1), 11-34. https://doi.org/10.1007/s11618-011-0175-6

UNESCO. (2005). Guidelines for inclusión: Ensuring acces to education for all. París: UNESCO.

Vonk, J. H. C. y Schras, G. A. (1987). From beginning to experienced teacher: A study of the professional development of teachers during their first four years of service. European Journal of Teacher Education, 1O(1), 95-1 10. https://doi.org/10.1080/0261976870100111

\section{Breve CV de las autoras}

\section{Tatiana Cisternas León}

Profesora de Educación Diferencial y Doctora en Ciencias de la Educación. Académica e investigadora de la Universidad Alberto Hurtado. Con experiencia en formación de profesores en diversas áreas vinculadas a la enseñanza en aulas diversas. A nivel de 
postgrado desarrolla formación en investigación cualitativa. Ha colaborado en diversas instancias de políticas públicas para la formación docente en inclusión. Desarrolla investigación y ha publicado en torno procesos de enseñanza, la formación inicial, la inserción de profesores noveles y el desarrollo profesional de docentes. ORCID ID: https://orcid.org/0000-0003-3142-0027. Email: tcistern@uahurtado.cl

\section{Amparo Lobos Gormaz}

Profesora de Educación Diferencial especialista en Problemas de Aprendizaje de la Universidad Metropolitana de Ciencias de la Educación. Docente de la Carrera de Educación Diferencial de la Universidad Alberto Hurtado en el área de la formación práctica. Se ha desempeñado como docente en contextos educativos formales y no formales participando en proyectos de alfabetización con niños y adultos mayores. Con experiencia de colaboración en investigación sobre docentes e interacciones en el aula. ORCID ID: https://orcid.org/o000-0001-9352-9349. Email: alobos@uahurtado.cl 